\title{
Differences in High Dose Antipsychotic Prescriptions in Patients with Schizophrenia in Asian Countries/Areas: Findings from the REAP-AP Study
}

\author{
Yong Chon Park', Shu-Yu Yang², Mian-Yoon Chong ${ }^{3}$, Shigenobu Kanba ${ }^{4}$, \\ Norman Sartorius ${ }^{5}$, Naotaka Shinfuku ${ }^{6}$, Chay-Hoon $\operatorname{Tan}^{7}$, and Seon-Cheol Park ${ }^{8} \bowtie$ \\ ${ }^{1}$ Department of Neuropsychiatry, Hanyang University Guri Hospital, Guri, Republic of Korea \\ ${ }^{2}$ Deparment of Pharmacy, Songde Branch, Tapei City Hospital, Tapei, Taiwan \\ ${ }^{3}$ Department of Psychiatry, Kaohsiung Chang Gung Memorial Hospital and Chang Gung University School of Medicine, Kaohsiung, Taiwan \\ ${ }^{4}$ Department of Neuropsychiatry, Graduate School of Medical Sciences, Kyushu University, Fukuoka, Japan \\ ${ }^{5}$ Association for the Improvement of Mental Health Programs, Geneva, Switzerland \\ ${ }^{6}$ Department of Social Welfare, School of Human Sciences, Seinan Gakuin University, Fukuoka, Japan \\ ${ }^{7}$ Department of Pharmacology, National University Hospital, Singapore \\ ${ }^{8}$ Department of Psychiatry, Inje University College of Medicine and Haeundae Paik Hospital, Busan, Republic of Korea
}

The REAP-AP study recruited 3,746 patients with schizophrenia, in March and April 2016, from 71 centers in 15 Asian countries/territories namely Bangladesh, China, Hong Kong, India, Indonesia, Japan, Korea, Malaysia, Myanmar, Pakistan, Singapore, Sri Lanka, Taiwan, Thailand and Vietnam. Our findings reveal a trend according to which high dose antipsychotic prescription is more prevalent in Eastern Asia (especially, Japan and Korea) than in other regions of Asia. This historical factor may be associated with our finding of an Eastern Asian preponderance of high dose antipsychotic prescription.

Psychiatry Investig 2018;15(10):1007-1008

Key Words Asian, Antipsychotic, High dose, Schizophrenia.

Although in the 1950s and the 1960s high dose antipsychotic treatment was regarded as effective and beneficial for schizophrenia, in the 1980s a dose-response relationship for antipsychotic treatment was found only in the range of 100 to $700 \mathrm{mg} /$ day of chlorpromazine equivalent dose. ${ }^{1}$ We aimed to analyze the Asian country/territory variation in high dose antipsychotic prescription in patients with schizophrenia, using the data from the Research on Asian Psychotropic Prescription Patterns for Antipsychotics (REAP-AP) study. ${ }^{2}$

The REAP-AP study recruited 3,744 patients with schizophrenia (F20), based on the International Classification of Disease, Tenth edition (ICD-10), ${ }^{3}$ in March and April 2016, from 71 centers in 15 Asian countries/areas namely Bangla-

\footnotetext{
Received: January 1, 2018 Accepted: September 6, 2018

$\square$ Correspondence: Seon-Cheol Park, MD, PhD

Department of Psychiatry, Inje University College of Medicine and Haeundae Paik Hospital, 875 Haeun-daero, Haeundae-gu, Busan 48108, Republic of Korea Tel: +82-51-797-3300, Fax: +82-51-797-0298

E-mail: cogito-ergo-sum@hanmail.net

(1) This is an Open Access article distributed under the terms of the Creative Commons Attribution Non-Commercial License (https://creativecommons.org/licenses/by$\mathrm{nc} / 4.0$ ) which permits unrestricted non-commercial use, distribution, and reproduction in any medium, provided the original work is properly cited.
}

desh, China, Hong Kong, India, Indonesia, Japan, Korea, Malaysia, Myanmar, Pakistan, Singapore, Sri Lanka, Taiwan, Thailand and Vietnam. The inclusion criteria were 1) diagnosis of schizophrenia, based on ICD- $10,{ }^{3}$ by clinical psychiatrists at the survey centers and 2) medication with antipsychotics and/or other psychotropic drugs. Informed consent was given by all study subjects prior to inclusion of the study, and the study protocol and informed consent forms were approved by institutional review boards of Tapei City Hospital, Tapei, Taiwan (receipt number: TCHIRB-10412128-E) and the other hospitals participating in survey. According to the Anatomical Therapeutic Chemical (ATC) classification system, ${ }^{4}$ antipsychotics (N05A) were defined. High dose antipsychotic prescription was defined as a ratio of prescribed daily dose (PDD) to defined daily dose (DDD) $>1.5$, or a cumulative dose per/day $>1,000 \mathrm{mg}$ of chlorpromazine equivalent dose.,

The mean frequency of high dose antipsychotic prescription by either definition in the subjects was $19.6 \%(n=734)$, and, in terms of PDD/DDD $>1.5$, the frequencies of high dose antipsychotic prescription in the different countries/areas ranged from $0.0 \%$ (Myanmar) to $26.2 \%$ (Japan) $\left(\chi^{2}=205.40, \mathrm{p}<0.001\right)$. 
In terms of cumulative dose, the frequency of high dose prescription ranged from $1.2 \%$ (Myanmar) to $34.5 \%$ (Japan) $\left(\chi^{2}=205.40, \mathrm{p}<0.001\right)$, and, in terms of PDD/DDD $>1.5$ or cu-

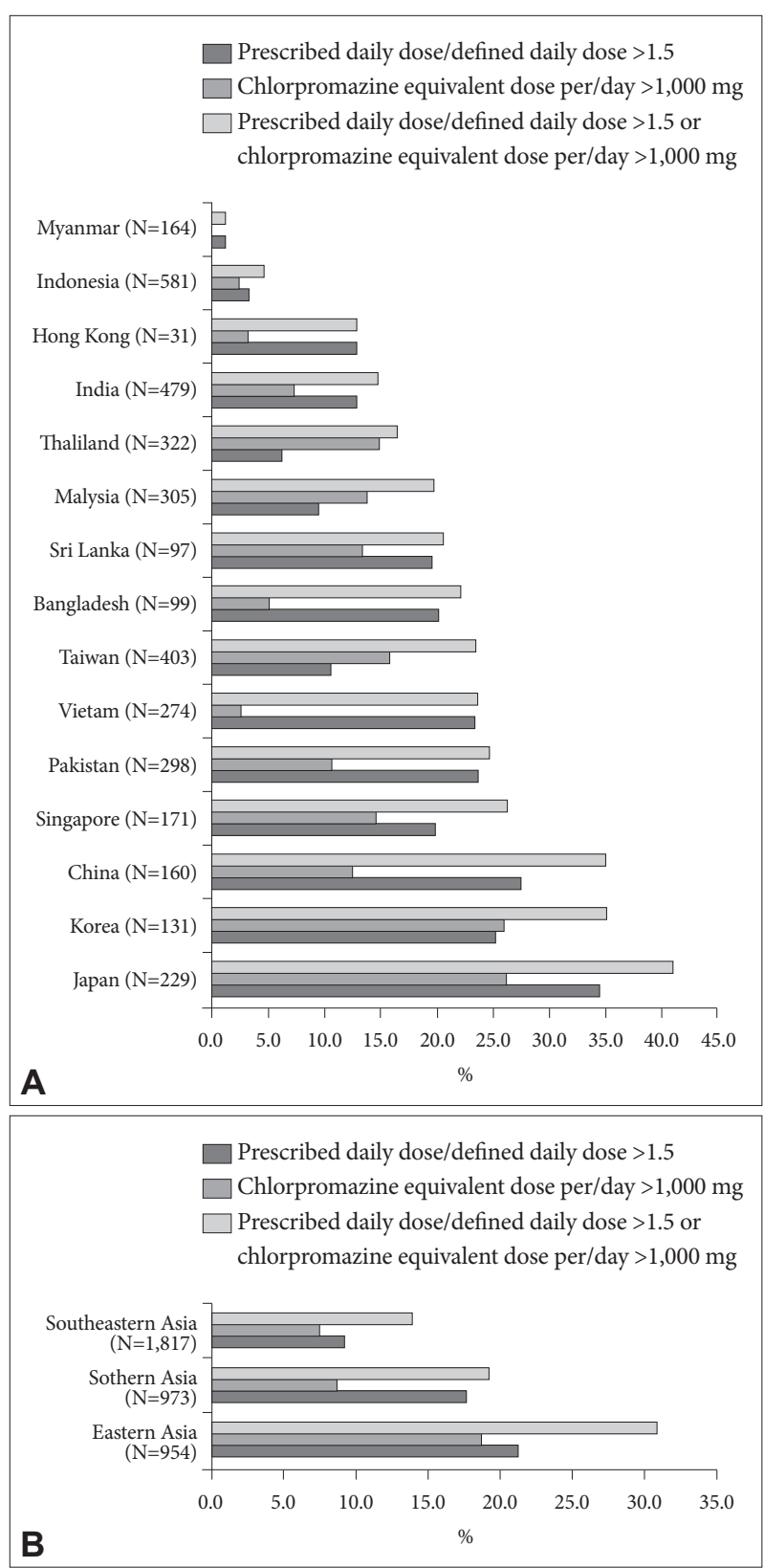

Figure 1. Asian country/territory variation in high dose antipsychotic prescriptions in patients with schizophrenia $(N=3,744)$. A: Frequencies of high dose antipsychotic prescriptions in different Asian countries/territories, using different definitions of high dose. $\mathrm{B}$ : High dose antipsychotic prescriptions in relation to regional classification of Asian countries/territories*. *Defined by United Nations classification: Eastern Asia (China, Hong Kong, Japan, Korea and Taiwan), Southern Asia (Bangladesh, India, Pakistan and Sri Lanka) and South-Eastern Asia (Indonesia, Malaysia, Myanmar, Singapore, Thailand and Vietnam). mulative dose $>1,000 \mathrm{mg} /$ day, the frequency ranged from $1.2 \%$ (Myanmar) to $41.0 \%$ (Japan) $\left(\chi^{2}=255.73, \mathrm{p}<0.001\right)$ (Figure 1A). In terms of the geographical classification of the different Asian countries/areas, using the definition of $\mathrm{PDD} / \mathrm{DDD}>1.5$, the frequency ranged from $7.5 \%$ (Southeastern Asia) to $18.7 \%$ (Eastern Asia) $\left(\chi^{2}=88.45, \mathrm{p}<0.001\right)$, while using the definition of cumulative dose $>1,000 \mathrm{mg} /$ day, the frequency ranged from 9.2\% (Southeastern Asia) to $21.3 \%$ (Eastern Asia) $\left(\chi^{2}=83.49, \mathrm{p}<\right.$ 0.001 ), and, using PDD/DDD $>1.5$ or a cumulative dose $>1,000$ $\mathrm{mg} /$ day, the frequency ranged from $13.9 \%$ (Southeastern Asia) to $30.9 \%$ (Eastern Asia) $\left(\chi^{2}=115.24, p<0.001\right)$ (Figure 1B).

Our findings reveal a trend according to which high dose antipsychotic prescription is more prevalent in Eastern Asia (especially, Japan and Korea) than in other regions of Asia. Psychopharmacotherapy can be greatly influenced by nonbiological, sociocultural and historical factors including drug costs, health insurance schemes, physicians' and/or patients' preferences etc. Historically, psychiatric institutions in Japan, Korea and other regions all shared the custom to use high and mega dose antipsychotic prescriptions to sedate unstable patients with schizophrenia. This historical factor may be associated with our finding of an Eastern Asian preponderance of high dose antipsychotic prescription. Interestingly, an association between high dose antipsychotic therapy and lower overall mortality has been revealed by a cohort study of 21,492 Swedish patients with schizophrenia. ${ }^{5}$ Further investigations of specific factors influencing high dose prescription and its clinical effectiveness are needed.

\section{Acknowledgments}

This research was supported by grants from Taipei City Hospital (1050162-012), Taipei, Taiwan.

\section{REFERENCES}

1. Suzuki T, Uchida H, Watanabe K, Yagi G, Kashima H. A clinical case series of switching from antipsychotic polypharmacy to montherapy with a second-generation agent on patients with chronic schizophrenia. Prog Neuropsychopharmacol Biol Psychiatry 2004;28:361-369.

2. Sim K, Su A, Leong IY, Yip K, Chong MY, Fujii S, et al. High dose antipsychotic use in schizophrenia: findings of the REAP (Research on East Asian Psychotropic Prescriptions) study. Pharmacopsychiatry 2004;37:175-179.

3. World Health Organization. The ICD-10 Classification of Mental and Behavioral Disorders, Clinical Descriptions and Diagnostic Guidelines. Geneva: World Health Organization; 1992.

4. World Health Organization. Anatomical Therapeutic Chemical (ATC) classification system. Available at: https://www.whocc.no/atc/structure_and_principles/. Accessed January 18, 2016.

5. Tihonen J, Mittendorfer-Rutz E, Tornianen M, Alexanderson K, Tanskane A. Mortality and cumulative exposure to antipsychotics, antidepressants, and benzodiazepine in patients with schizophrenia: an observational follow-up study. Am J Psychiatry 2016;173:600-606. 